Revue d'histoire de l'Amérique française

7S REVUE D.HISTOIRE DE L'AMÉRIQUE FRANÇAISE

\title{
Crise ou modernisation. La société rurale maskoutaine durant le premier tiers du XIX ${ }^{\mathrm{e}}$ siècle
}

\section{Christian Dessureault}

Volume 42, numéro 3, hiver 1989

URI : https://id.erudit.org/iderudit/304706ar

DOI : https://doi.org/10.7202/304706ar

Aller au sommaire du numéro

\section{Éditeur(s)}

Institut d'histoire de l'Amérique française

\section{ISSN}

0035-2357 (imprimé)

1492-1383 (numérique)

Découvrir la revue

\section{Citer cet article}

Dessureault, C. (1989). Crise ou modernisation. La société rurale maskoutaine durant le premier tiers du XIX ${ }^{\mathrm{e}}$ siècle. Revue d'histoire de l'Amérique française, 42(3), 359-387. https://doi.org/10.7202/304706ar
Résumé de l'article

Crise ou modernisation? L'évolution de la société rurale bas-canadienne durant le premier tiers du $\mathrm{XIX}^{\mathrm{e}}$ siècle suscite beaucoup de controverses. Les objectifs du présent article sont de vérifier, à l'échelle micro-régionale, la croissance ou le recul de l'économie rurale et de caractériser les structures économiques et sociales au cours de cette période. L'évolution des campagnes maskoutaines s'insère dans une dynamique de transition au capitalisme. La pénétration croissante du marché entraîne l'amélioration globale des niveaux de vie et des niveaux de fortune. Elle favorise aussi la différenciation sociale de la paysannerie. L'émergence du prolétariat rural et de l'artisanat résultent à la fois de la croissance économique et de la poussée démographique. Les transformations de la société rurale ne sont toutefois ni rapides ni radicales. La société rurale est au rendez-vous de la croissance économique durant le premier tiers du XIX ${ }^{\mathrm{e}}$ siècle. Mais ce type de développement qui renforce les inégalités au sein de la société rurale ne modifie pas substantiellement les anciennes structures économiques et sociales.
Tous droits réservés @ Institut d'histoire de l'Amérique française, 1989
Ce document est protégé par la loi sur le droit d'auteur. L'utilisation des services d'Érudit (y compris la reproduction) est assujettie à sa politique d'utilisation que vous pouvez consulter en ligne.

https://apropos.erudit.org/fr/usagers/politique-dutilisation/ 


\title{
CRISE OU MODERNISATION. LA SOCIÉTÉ RURALE MASKOUTAINE DURANT LE PREMIER TIERS DU XIXe SIÉCLE ${ }^{1}$
}

\author{
CHRISTIAN DESSUREAULT \\ Département d' histoire \\ Université de Montréal
}

\begin{abstract}
RÉSUMÉ
Crise ou modernisation? L'évolution de la société rurale bas-canadienne durant le premier tiers du XIXe siècle suscite beaucoup de controverses. Les objectifs du présent article sont de vérifier, à l'échelle micro-régionale, la croissance ou le recul de l'économie rurale et de caractériser les structures économiques et sociales au cours de cette période. L'évolution des campagnes maskoutaines s'insère dans une dynamique de transition au capitalisme. La pénétration croissante du marché entraîne l'amélioration globale des niveaux de vie et des niveaux de fortune. Elle favorise aussi la différenciation sociale de la paysannerie. L'émergence du prolétariat rural et de l'artisanat résultent à la fois de la croissance économique et de la poussée démographique. Les transformations de la société rurale ne sont toutefois ni rapides ni radicales. La société rurale est au rendez-vous de la croissance économique durant le premier tiers du XIXe siècle. Mais ce type de développement qui renforce les inégalités au sein de la société rurale ne modifie pas substantiellement les anciennes structures économiques et sociales.
\end{abstract}

\begin{abstract}
Controversy over whether Lower Canada was undergoing a crisis or a process of modernization has surrounded the study of the evolution of rural society during the first third of the nineteenth century. This article proposes to analyse the rural economy and to describe social and economic structures on a micro-regional level. The countryside around Saint-Hyacinthe evolved within the framework of the transition to capitalism. The growing penetration of the market led to increased living standards and wealth. It also caused greater social differentiation among the peasantry. The emergence of a rural proletariat and artisanal class resulted from both economic and demographic growth. Changes in rural society were not, however, rapid or radical. Economic growth which caracterized the whole period reinforced inequality within rural society but did not substantially modify the dominant economic and social structures.
\end{abstract}

Crise ou modernisation? L'évolution des structures économiques et sociales des campagnes bas-canadiennes durant le premier tiers du XIXe siècle a suscité et suscite encore beaucoup de controverses. Les

\footnotetext{
1 Nous tenons particulèrement à remercier les professeurs Louis Michel et Pierre Tousignant, du département d'histoire de l'Université de Montréal, ainsi que Lise St-Georges, étudiante au doctorat au même département, qui ont patiemment lu et commenté la première version du
} texte. 
historiens avaient déjà souligné les malaises de l'agriculture bas-canadienne avant que Jean Hamelin et Fernand Ouellet présentent, en 1962, la thèse d'une crise structurelle de la société rurale au cours de cette période $^{2}$. Par la suite, Fernand Ouellet systématise ce modèle de crise de la société rurale et le blocage agraire en devient l'une des composantes majeures ${ }^{3}$. Cette crise entraîne le durcissement des structures sociales traditionnelles et le repli vers une agriculture d'autosubsistance. Elle se concrétise également par l'émergence d'un prolétariat rural, l'appauvrissement de la paysannerie et, par voie de conséquence, des notables ruraux.

$\mathrm{Au}$ cours des dernières années, plusieurs travaux ont ébranlé l'édifice sur lequel repose la crise agricole. Cependant, seuls Gilles Paquet et Jean-Pierre Wallot ont proposé, dès 1971, un modèle d'interprétation global pour saisir la dynamique du système économique et social bascanadien au début du XIXe siècle, soit le concept de modernisation ${ }^{4}$. Le commerce leur apparaît l'élément moteur de l'économie et ils refusent explicitement le postulat «labroussien» selon lequel le secteur agricole influe de manière déterminante sur l'ensemble de l'économie'. Néanmoins, loin de demeurer à l'écart du nouveau souffle de croissance, les campagnes bénéficient grandement de la pénétration rapide de l'économie de marché: accroissement des niveaux de fortune, rationalisation de la production, diffusion de la consommation ${ }^{6}$. Le concept de modernisation englobe non seulement l'idée d'une croissance économique, mais aussi celle d'une rupture radicale ou d'une mutation des structures économiques et sociales.

Dans ses travaux récents, Serge Courville apporte plusieurs arguments favorables à la modernisation du Bas-Canada durant cette période $^{7}$. La croissance villageoise, particulièrement forte entre 1815 et 1831, traduit la montée du capitalisme. «D'abord comme place de

\footnotetext{
2 Jean Hamelin et Fernand Ouellet, «La crise agricole dans le Bas-Canada, 1802-1837», Études rurales, 3,4 (1962): 37-57.

3 F. Ouellet, «Le mythe de l'habitant sensible au marché. Commentaires sur la controverse Le Goff - Wallot et Paquet», Recherches sociographiques, 17 (1975): 115-132; F. Ouellet, Le Bas-Canada 1791-1840. Changements structuraux et crise (Ottawa, Éditions de l'Université d'Ottawa, 1980).

4 Gilles Paquet et Jean-Pierre Wallot, «Le Bas-Canada au début du XIXe siècle: une hypothèse», Revue d' histoire de l'Amérique française, 25,1 (juin 1971): 39-61.

5 Ibidem, «Crise agricole et tensions socio-ethniques dans le Bas-Canada, 1802-1812: éléments pour une ré-interprétation», $R H A F, 26,2$ (mars 1972): 185-237.

6 Ibidem, «Les inventaires après décès à Montréal au tournant du XIXe siècle: préliminaires à une analyse», RHAF, 30,2 (septembre 1976): 163-221; «Structures sociales et niveaux de richesse dans les campagnes du Québec, 1792-1812», Bulletin d'histoire de la culture matérielle, 17 (1983): 25-44.

Serge Courville, «Esquisse du développement villageois au Québec: le cas de l'aire seigneuriale entre 1760 et 1851», Cahiers de géographie du Québec, 28,73-74 (1984): 9-46; «Croissance villageoise et industries rurales dans les seigneuries du Québec (1815-1851)», François Lebrun et Normand Séguin, Sociétés villageoises et rapports villes-campagnes au Québec et dans la France de l'Ouest XVIIe-XXe siècles (CREQ-UQTR, Trois-Rivières, 1987), 205-220.
} 
marché, mais aussi et très tôt comme lieux de production et de fabrication artisanale et manufacturière (...) ceux-ci (les villages) sont des lieux de cristallisation des industries rurales. ${ }^{8}$ Ces villages constituent en quelque sorte les témoins d'une pré-industrialisation des campagnes. Celles-ci sont désormais à l'avant-garde du procès de modernisation des structures économiques et sociales et, ce faisant, les industries rurales se substituent à l'agriculture comme secteur moteur de l'économie rurale.

Les objectifs du présent article sont à la fois ambitieux et modestes. Nous voulons scruter, à l'échelle micro-régionale, l'évolution de certains aspects de la structure économique et sociale des campagnes durant le premier tiers du XIXe siècle. Globalement, l'évolution des campagnes maskoutaines s'insère dans une dynamique de transition au capitalisme. La pénétration croissante de l'économie de marché entraîne l'amélioration globale des niveaux de vie et des niveaux de fortune. Elle favorise aussi la différenciation sociale au sein de la paysannerie. L'émergence du prolétariat rural et l'essor de l'artisanat rural résultent à la fois de la croissance économique et de la poussée démographique dans les campagnes. Cette dernière accélère la mutation des structures économiques et sociales, mais les moteurs véritables du changement social sont déjà inscrits dans la dynamique de l'économie rurale. Crise sociale et croissance économique ne sont pas deux voies contradictoires de l'histoire des campagnes.

Les transformations du monde rural ne sont toutefois ni rapides ni radicales. Les campagnes des années 1830 conservent de nombreux traits de la société ancienne. L'agriculture demeure le secteur d'activité économique prédominant et les ruraux qui ne vivent pas directement de l'agriculture en demeurent encore largement tributaires. Certaines mutations sociales du premier tiers du XIXe siècle résultent de l'accentuation des contradictions internes de la société rurale. L'émergence du prolétariat rural représente l'aboutissement des inégalités économiques et sociales de la paysannerie. Le fossé des inégalités qui se creuse à la campagne contribue à la transformation de l'ensemble du système.

À l'aide du recensement de 1831, nous mesurons d'abord le poids respectif des divers secteurs d'activité dans l'économie rurale. Les statuts professionnels fournissent également un premier aperçu de la hiérarchie sociale. Cette enquête apporte aussi plusieurs séries de données, dans le temps et dans l'espace, sur l'évolution de la propriété foncière et des fortunes mobilières. Ces données s'avèrent des indices pertinents pour évaluer la croissance ou le recul de l'économie rurale et pour caractériser la structure économique et sociale des campagnes maskoutaines durant le premier tiers du XIXe siècle. La terre demeure le principal bien de production et l'accès à la propriété foncière représente

$8 \quad$ Ibidem, «Croissance villageoise...», 211. 


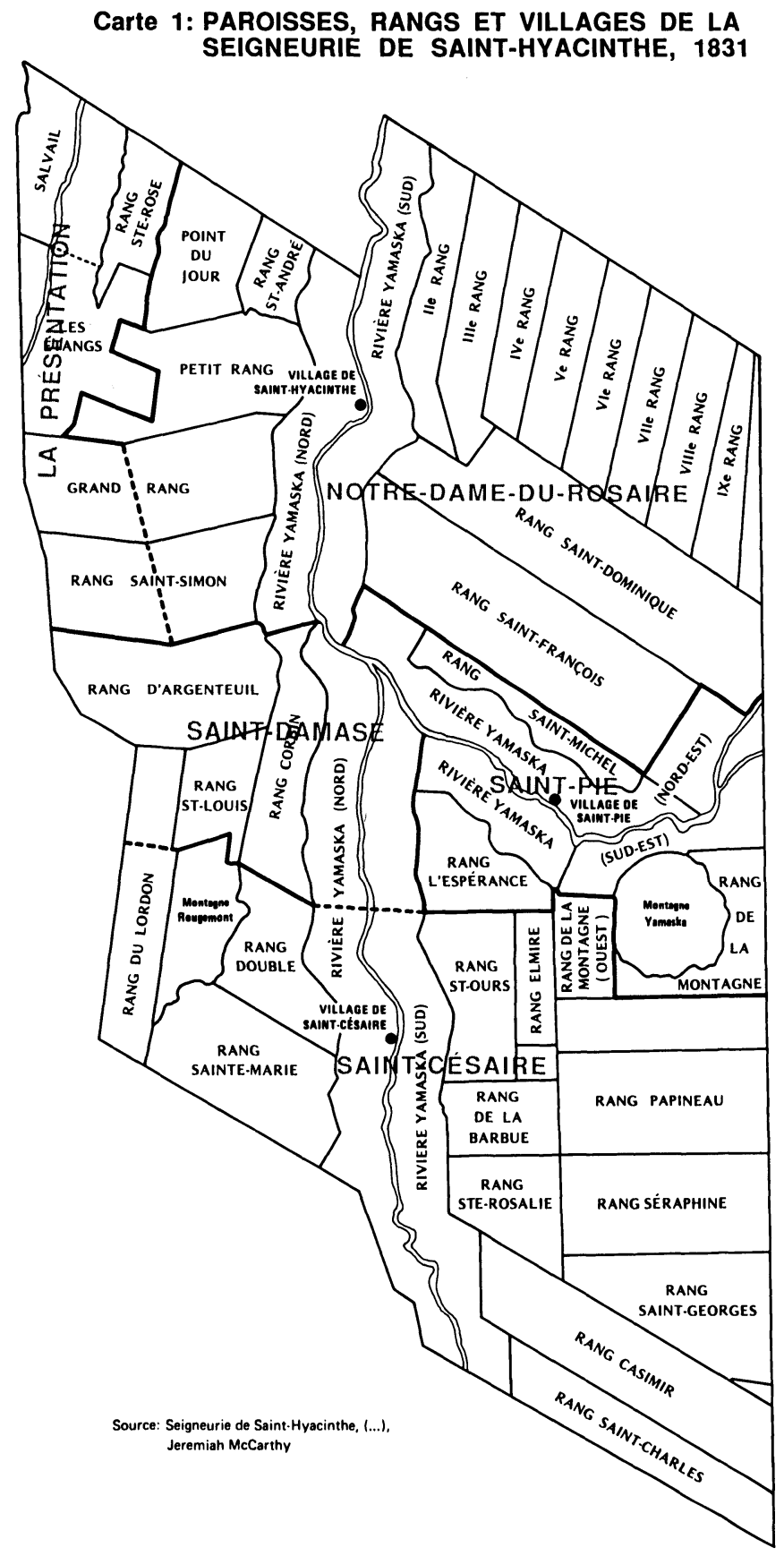


l'un des enjeux majeurs de la vie paysanne. Cependant, à défaut d'une évaluation des biens fonciers, la fortune mobilière constitue un meilleur indice du statut socio-économique que la superficie de la propriété et, surtout, elle permet de comparer les niveaux de richesse des divers groupes sociaux.

\section{1 - LE TERRAIN D'ENQUETTE}

Cette enquête est circonscrite à la seigneurie primitive de SaintHyacinthe (voir carte 1). Au début du XIXe siècle, la paroisse de NotreDame-du-Rosaire de Saint-Hyacinthe englobe l'ensemble du territoire de cette seigneurie. En 1806, les autorités procèdent à la création d'une seconde paroisse, La Présentation. Au début des années 1820, la croissance démographique entraîne la formation de deux nouvelles paroisses

TABLEAU 1

Évolution de la population dans la seigneurie de Saint-Hyacinthe, 1821-1831

\begin{tabular}{|c|c|c|c|c|}
\hline Années & Population & Intervalle & $\begin{array}{c}\text { Croissance } \\
\text { naturelle }\end{array}$ & $\begin{array}{c}\text { Solde } \\
\text { migratoire }\end{array}$ \\
\hline 1821 & 10051 & & & \\
1825 & 11742 & $1821-1825$ & 1306 & +385 \\
1831 & 14098 & $1825-1831$ & 2864 & -508 \\
\hline
\end{tabular}

Sources: JCABC, 1821/1822. ANC, Recensement de 1825, comtés de Richelieu et de Bedford, bobine C-717. ANC, Recensement de 1831, comté de Saint-Hyacinthe, bobine C-722. ANQM, Registres d'état civil des paroisses de La Présentation, de Notre-Dame-duRosaire, de Saint-Hyacinthe, de Saint-Césaire, de Saint-Damase et de Saint-Pie.

\section{TABLEAU 2}

Ventilation par paroisse de l'évolution de la population dans la seigneurie de Saint-Hyacinthe, 1825-1831

\begin{tabular}{|l|cc|c|c|}
\hline & \multicolumn{2}{|c|}{ Population } & \multicolumn{1}{c|}{$\begin{array}{c}\text { Croissance } \\
\text { naturelle }\end{array}$} & \begin{tabular}{c} 
Solde \\
migratoire \\
\multicolumn{1}{|c|}{ Paroisse }
\end{tabular} \\
\hline 1825 & 1831 & $1825-1831$ & $1825-1831$ \\
\hline La Présentation & 1712 & 1646 & 229 & -295 \\
$\begin{array}{l}\text { Notre-Dame-du-Rosaire } \\
\text { et Saint-Pie }\end{array}$ & 6600 & $-7611^{*}$ & 1546 & -535 \\
Saint-Césaire & 1725 & 2796 & 612 & +459 \\
Saint-Damase & 1705 & 2045 & 477 & -137 \\
\hline
\end{tabular}

* En 1831, Notre-Dame-du-Rosaire et Saint-Pie comptent respectivement 5317 et 2294 habitants.

Sources: Voir tableau 1. 
au sud de la seigneurie: Saint-Césaire et Saint-Damase. En 1830, NotreDame-du-Rosaire subit une autre division lors de l'établissement de Saint-Pie dans le secteur est, aux environs de la montagne Yamaska. Vers 1830, la seigneurie de Saint-Hyacinthe compte cinq paroisses auxquelles viendront se greffer deux nouvelles paroisses avant la fin de la décennie: Sainte-Rosalie et Saint-Dominique, qui seront tour à tour détachées de la paroisse mère de Notre-Dame-du-Rosaire.

Cette seigneurie, d'environ $825 \mathrm{~km}^{2}$, située au sud-est de la plaine de Montréal, est l'un des terroirs les plus fertiles du Québec. Les terres sablo-argileuses des pourtours de la rivière Yamaska sont particulièrement favorables aux cultures céréalières qui dominaient le paysage agraire durant le premier tiers du XIXe siècle. Certes, la qualité des sols n'est pas totalement uniforme. Ainsi, à La Présentation, les paysans de la rivière Salvail et des Étangs disposent de terres plutôt humides, parsemées de petits marécages qui conviennent davantage à l'exploitation de prairies naturelles qu'aux cultures céréalières. Néanmoins, le type de sols de la plaine maskoutaine, commun aux cinq paroisses de la seigneurie, devrait plutôt favoriser l'essor économique de la paysannerie.

$\mathrm{Au}$ début du XIXe siècle, la seigneurie est une région de peuplement récent qui accueille bon an mal an des contingents de nouveaux colons. En 1791, elle compte seulement 1360 habitants ${ }^{9}$. Quinze ans plus tard, la population se situe autour de 5000 habitants, et la forte croissance démographique se maintient au début du XIXe siècle pour atteindre 10051 habitants en 1821 et 14098 en $1831^{10}$. Toutefois, à partir de 1825, la seigneurie n'absorbe plus l'ensemble de ses excédents démographiques. L'espace agraire disponible se raréfie et la concurrence devient plus vive pour l'acquisition de nouvelles terres.

À l'instar de la plaine environnante, le village de Saint-Hyacinthe se développe rapidement durant le premier tiers du XIXe siècle. De 1805 à 1831, sa population passe de 321 à 914 habitants, ce qui le place au troisième rang dans l'ensemble des agglomérations villageoises du Bas-Canada $^{11}$. Le recensement de 1831 identifie deux autres villages dans la seigneurie de Saint-Hyacinthe, soit Saint-Césaire, nommé également Burtonville, avec 236 habitants et Saint-Pie, avec 195 habitants ${ }^{12}$. L'émergence de ces noyaux villageois est toute récente puisqu'ils sont

9 Recensements du Canada, vol. 4.

10 Voir les tableaux 1 et 2.

11 Soit après les villages de Laprairie et de William Henry (Sorel). Pour des données précises sur la population des villes et des principaux villages du Bas-Canada en 1831, voir JeanClaude Robert, Montréal 1821-1871. Aspects de l'urbanisation, thèse de doctorat de 3e cycle, Paris, 102. Voir aussi C.-P. Choquette, Histoire de la ville de Saint-Hyacinthe, 29; ANC, Recensement de 1831, comté de Saint-Hyacinthe, bobine C-722.

12 ANC, Recensement de 1831, comté de Saint-Hyacinthe, bobine C-722. 
situés au centre des nouveaux secteurs de peuplement de la seigneurie. Le village de Saint-Damase, une aire d'une quinzaine d'emplacements dans le rang Corbin, est encore à l'état embryonnaire.

\section{2 - LES STRUCTURES SOCIO-PROFESSIONNELLES AU DÉBUT DES ANNÉES 1830}

Le recensement de 1831 fournit le nom et la profession des chefs de ménage. La qualité des déclarations n'est toutefois pas uniforme dans tous les comtés et, à l'intérieur d'un même comté, on observe certaines variations dans l'enregistrement des professions selon les paroisses. Le traitement agrégé de ces déclarations, à l'échelle du BasCanada, demeure une entreprise hasardeuse. Ainsi, dans certains comtés, les journaliers ne sont pas identifiés comme tels et il est difficile de les repérer dans la vaste catégorie de profession inconnue. À Saint-Hyacinthe, le recenseur a été heureusement plus explicite dans l'attribution des professions des chefs de ménage masculins. Mais, en ce qui concerne les chefs de ménage féminins, les omissions sont fréquentes.

Il y a toutefois des anomalies d'une paroisse à l'autre. Ainsi le recensement dénombre davantage de chefs de ménage «rentiers» dans les terroirs de peuplement récent de Saint-Césaire, de Saint-Damase et de Saint-Pie que dans les terroirs plus anciens de La Présentation et de Notre-Dame-du-Rosaire. Cela apparaît peu vraisemblable. Dans ces deux dernières paroisses, plusieurs cultivateurs sans propriété foncière sont évidemment des «rentiers» non identifiés comme tels.

Comme le soulignent plusieurs historiens ${ }^{13}$, l'utilisation des mentions professionnelles des recensements ou d'autres sources de même type soulève toujours des problèmes. Nos données sur la structure socioprofessionnelle n'ont donc pas la prétention de saisir précisément la dynamique des activités économiques et de dévoiler la hiérarchie de la société maskoutaine au début des années 1830 . Néanmoins, elles font ressortir certains grands traits de cette société rurale qu'il sera possible d'affiner grâce aux inventaires après décès.

La répartition par groupes socio-professionnels des chefs de ménage donne un premier aperçu de l'économie maskoutaine en 1831 (voir les tableaux 3 et 4). Apparemment, l'agriculture demeure le secteur dominant de l'économie locale, celui auquel est directement liée la grande majorité des chefs de ménage. Le nombre de ceux-ci engagés dans la fabrication et la construction est relativement faible et ils sont concentrés surtout dans les villages.

13 Jean-Paul Bernard et al., «La structure professionnelle de Montréal en 1825», RHAF, 30,3 (décembre 1976): 383-415; Gérard Bouchard et al., «Le classement des professions par secteurs d'activité: aperçu critique et présentation d'une nouvelle grille», L'Actualité Économique (oct.-déc. 1979): 585-605; Louise Dechêne, «Quelques aspects de la ville de Québec au XVIIIe siècle d'après les dénombrements paroissiaux», Cahiers de géographie du Québec, 28,75 (1984): 485-505. 
TABLEAU 3

La structure socio-professionnelle dans les paroisses maskoutaines, 1831

(d'après la profession des chefs de ménages)

\begin{tabular}{|c|c|c|c|c|c|c|c|c|c|c|c|c|}
\hline \multirow{2}{*}{$\begin{array}{l}\text { Groupes socio- } \\
\text { professionnels }\end{array}$} & \multicolumn{2}{|c|}{ Saint-Hyacinthe } & \multicolumn{2}{|c|}{ La Présentation } & \multicolumn{2}{|c|}{ Saint-Damase } & \multicolumn{2}{|c|}{ Saint-Césaire } & \multicolumn{2}{|c|}{ Saint-Pie } & \multicolumn{2}{|c|}{ Total } \\
\hline & Nombre & $\%$ & Nombre & $\%$ & Nombr & $\%$ & Nombre & $\%$ & Nombre & $\%$ & Nombre & $\%$ \\
\hline 1. Seigneur & 1 & 0,1 & - & & - & & - & & - & & 1 & - \\
\hline 2. Clercs & 3 & 0,4 & 1 & 0,3 & 1 & 0,3 & 1 & 0,2 & 2 & 0,5 & 8 & 0,3 \\
\hline 3. Professions libérales & 5 & 0,6 & - & & - & & 3 & 0,6 & 2 & 0,5 & 10 & 0,4 \\
\hline 4. Bourgeois & 1 & 0,1 & - & & - & & - & & - & & 1 & - \\
\hline 5. Marchands & 17 & 2,0 & 3 & 1,1 & 1 & 0,3 & 4 & 0,8 & 2 & 0,5 & 27 & 1,1 \\
\hline 6. Aubergistes & 5 & 0,6 & - & & - & & 3 & 0,6 & 2 & 0,5 & 10 & 0,5 \\
\hline 7. Fonctionnaires & 3 & 0,4 & 1 & 0,3 & - & & 1 & 0,2 & 1 & 0,2 & 6 & 0,3 \\
\hline 8. Artisans & 110 & 12,7 & 11 & 4,0 & 21 & 6,2 & 42 & 8,3 & 34 & 7,9 & 218 & 9,1 \\
\hline a) Construction & \multicolumn{2}{|c|}{29} & \multicolumn{2}{|c|}{5} & \multicolumn{2}{|c|}{6} & \multicolumn{2}{|c|}{14} & \multicolumn{2}{|c|}{14} & \multicolumn{2}{|c|}{68} \\
\hline b) Fabrication & \multicolumn{2}{|c|}{75} & \multirow{2}{*}{\multicolumn{2}{|c|}{ 6 }} & \multicolumn{2}{|c|}{15} & \multirow{2}{*}{\multicolumn{2}{|c|}{28}} & \multicolumn{2}{|c|}{20} & \multicolumn{2}{|c|}{144} \\
\hline c) Transport & \multicolumn{2}{|c|}{6} & & & & & & & & & \multicolumn{2}{|c|}{6} \\
\hline 9. Cultivateurs & 488 & 56,6 & 189 & 68,5 & 227 & 67,0 & 361 & 71,6 & 280 & 65,5 & 1545 & 64,2 \\
\hline 10. Fermiers & 50 & 5,8 & 19 & 6,9 & 32 & 9,4 & 7 & 1,4 & 23 & 5,4 & 131 & 5,4 \\
\hline 11. Journaliers & 132 & 15,4 & 40 & 14,5 & 36 & 10,6 & 58 & 11,5 & 55 & 12,9 & 321 & 13,3 \\
\hline 12. Métiers divers & 8 & 0,3 & 3 & 1,1 & 1 & 0,3 & 8 & 1,6 & 8 & 1,9 & 28 & 1,2 \\
\hline 13. Rentiers & 3 & 0,4 & - & & 11 & 3,2 & 14 & 2,8 & 14 & 3,3 & 42 & 1,3 \\
\hline 14. Veuves & 30 & 3,5 & 9 & 3,3 & 8 & 2,4 & 1 & 0,2 & 4 & 0,9 & 52 & 2,2 \\
\hline 15. Mendiants & 5 & 0,6 & - & & 1 & 0,3 & 1 & 0,2 & - & & 7 & 0,3 \\
\hline \multirow{2}{*}{$\begin{array}{l}\text { 16. Indéfini } \\
\text { TOTAL }\end{array}$} & 1 & 0 & - & & - & & - & & - & & 1 & - \\
\hline & 862 & 100,0 & 276 & 100,0 & 339 & 100,0 & 504 & 100,0 & 427 & 100,0 & 2408 & 100,0 \\
\hline
\end{tabular}

Source: Recensement de 1831. 
TABLEAU 4

La structure socio-professionnelle dans les villages maskoutains, 1831 (d'après la profession des chefs de ménages)

\begin{tabular}{|c|c|c|c|c|c|c|c|c|}
\hline \multirow{2}{*}{$\begin{array}{l}\text { Groupes socio- } \\
\text { professionnels }\end{array}$} & \multicolumn{2}{|c|}{ Saint-Hyacinthe } & \multicolumn{2}{|c|}{ Saint-Césaire } & \multicolumn{2}{|c|}{ Saint-Pie } & \multicolumn{2}{|c|}{ Total } \\
\hline & Nombre & $\%$ & Nombre & $\%$ & Nombre & $\%$ & Nombre & $\%$ \\
\hline 1. Seigneur & 1 & 0,6 & - & & - & & 1 & 0,4 \\
\hline 2. Clercs & 3 & 1,9 & 1 & 2,0 & 1 & 2,3 & 5 & 2,0 \\
\hline 3. Professions & & & & & & & & \\
\hline libérales & 4 & 2,5 & 3 & 5,9 & 1 & 2,3 & 8 & 3,1 \\
\hline 4. Bourgeois & - & - & - & & - & & - & \\
\hline 5. Marchands & 12 & 7,5 & 4 & 7,8 & 1 & 2,3 & 17 & 6,7 \\
\hline 6. Aubergistes & 4 & 2,5 & 3 & 5,9 & 1 & 2,3 & 8 & 3,1 \\
\hline 7. Fonctionnaires & 4 & 2,5 & 1 & 2,0 & - & & 5 & 2,0 \\
\hline 8. Artisans & 50 & 31,2 & 23 & 45,1 & 13 & 30,2 & 86 & 33,9 \\
\hline a) Construction & 12 & & 6 & & 5 & & 23 & \\
\hline b) Fabrication & 33 & & 17 & & 8 & & 58 & \\
\hline c) Transport & 5 & & - & & - & & 5 & \\
\hline 9. Cultivateurs & 9 & 5,6 & - & & 3 & 7,0 & 12 & 4,7 \\
\hline 10. Fermiers & - & - & - & & - & & - & - \\
\hline 11. Journaliers & 60 & 37,5 & 8 & 15,6 & 16 & 37,3 & 84 & 33,1 \\
\hline 12. Métiers divers & 1 & 0,6 & 3 & 5,9 & 2 & 4,7 & 6 & 2,4 \\
\hline 13. Rentiers & - & - & 5 & 9,8 & 5 & 11,6 & 10 & 3,9 \\
\hline 14. Veuves & 10 & 6,3 & - & & - & & 10 & 3,9 \\
\hline 15. Mendiants & 2 & 1,3 & - & & - & & 2 & 0,8 \\
\hline 16. Indéfini & - & - & - & & - & & - & \\
\hline TOTAL & 160 & 100, & 51 & 100 & 43 & 100 & 254 & 100 \\
\hline
\end{tabular}

Source: Recensement de 1831.

Le village de Saint-Hyacinthe compte une cinquantaine d'artisans parmi ses 160 chefs de ménage, dont 33 dans le secteur de la fabrication, 12 dans celui de la construction et 5 dans celui du transport. Dans le secteur de la fabrication, les principales concentrations d'activité sont la production alimentaire avec 11 chefs de ménage, dont 5 boulangers et 3 bouchers, et l'industrie artisanale du cuir avec 7 cordonniers et 3 tanneurs. À cet égard, le village de Saint-Hyacinthe n'apparait guère comme le catalyseur d'une proto-industrialisation de la campagne maskoutaine ${ }^{14}$. Les villages de Saint-Pie et de Saint-Césaire ont leurs petits noyaux d'artisans, parmi lesquels un groupe de six cordonniers et un tanneur à Saint-Césaire.

L'analyse détaillée des déclarations de profession, à l'échelle de la seigneurie, suggère la dimension embryonnaire du secteur de la fabrication et ses liens souvent étroits avec le secteur agricole. Dans la partie rurale de la seigneurie, les sept chaufourniers du rang Saint-Dominique

14 Sur le village comme catalyseur de l'industrialisation naissante des campagnes, voir S. Courville, «Croissance villageoise...». 
constituent le seul noyau artisanal quelque peu important, mais six d'entre eux sont des cultivateurs possédant une terre de 60 arpents et plus. La grande majorité des autres artisans qui résident dans les campagnes exerce des métiers liés à l'agriculture, tels les charrons et les forgerons ${ }^{15}$. Cet artisanat de service répond à la fois à la croissance de la population et au développement du secteur agricole.

Les journaliers représentent un peu plus de $13 \%$ des chefs de ménage maskoutains. Afin de caractériser la valence des secteurs d'activité dans l'économie maskoutaine, il importe de situer leur fonction principale. S'agit-il d'abord de travailleurs agricoles ou d'une maind'oeuvre principalement engagée dans l'industrie rurale embryonnaire ${ }^{16}$ ?

La concentration de journaliers dans les villages, en particulier au bourg de Saint-Hyacinthe, suggère l'existence d'une nombreuse maind'oeuvre libérée de l'agriculture. Toutefois, divers indices convergent dans l'autre direction. La paroisse la plus rurale et la plus agricole de la seigneurie, La Présentation, compte une proportion presque aussi forte de journaliers que la paroisse plus «urbanisée» de Notre-Damedu-Rosaire et une proportion plus élevée que celles Saint-Césaire et de Saint-Pie, où existent des noyaux villageois. À l'échelle de la seigneurie, les journaliers sont plutôt disséminés dans l'espace de manière à répondre aux besoins du secteur agricole (voir carte 2). Enfin, si les villages sont pour les journaliers des foyers d'activités non agricoles, que ce soit comme main-d'oeuvre d'appoint dans les secteurs du commerce, de la construction, de la fabrication ou du transport, les journaliers de la zone rurale déclarent fort majoritairement vivre d'abord de l'agriculture lors du recensement de 1831 (voir tableau 5).

Cette analyse souligne bien la prépondérance de l'agriculture dans l'univers de la production. Les autres secteurs économiques accaparent encore peu de main-d'oeuvre et demeurent encore fortement liés à l'agriculture. Par ailleurs, les bourgs sont certainement les foyers principaux de la fabrication artisanale et, au mieux, les noyaux d'une petite industrie rurale embryonnaire. Mais ils sont également, ce qui n'est pas négligeable, les lieux où se concentrent le commerce, les services professionnels, les pouvoirs religieux et seigneuriaux, numériquement moins importants que l'artisanat rural, mais dont le poids économique et social sur la campagne environnante est beaucoup plus considérable. Ainsi, on trouve dans le village de Saint-Hyacinthe une forte concen-

\footnotetext{
15 Par ailleurs, le tiers des familles d'artisans résidant dans la zone rurale de la seigneurie gagnaient leur subsistance par des travaux d'agriculture selon le recensement de 1831. Ainsi, plusieurs charrons et forgerons de campagne sont à la fois artisans et cultivateurs.

${ }_{16} \mathrm{~F}$. Ouellet soutient la première hypothèse: «Répartition de la propriété foncière et types d'exploitation agricole dans la seigneurie de Laprairie durant les années 1830», Éléments d'histoire sociale du Bas-Canada (Montréal, Hurtubise HMH, 1972), 110-119; S. Courville soutient la seconde: «Croissance villageoise...».
} 


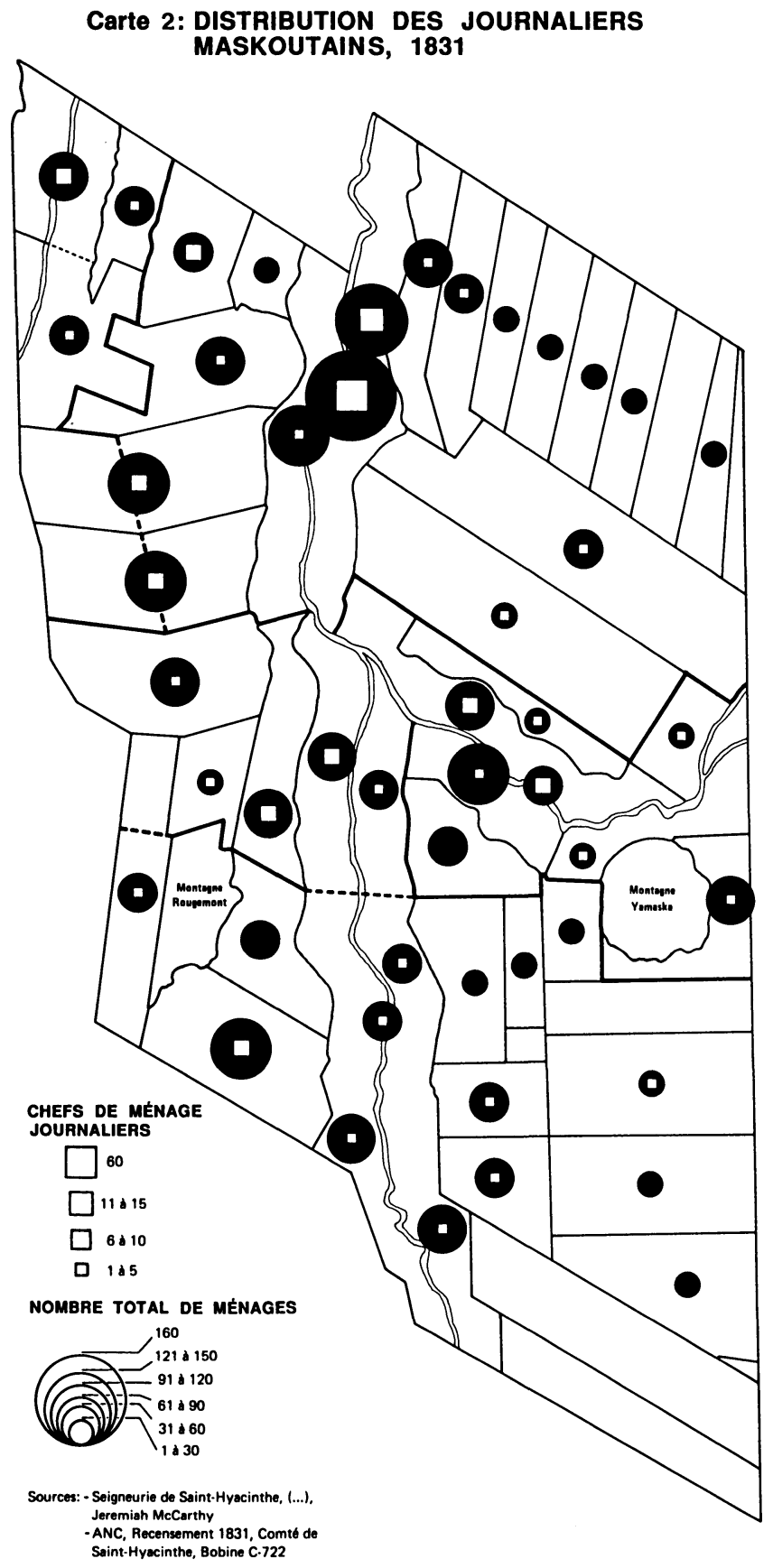




\section{TABLEAU 5}

Distribution des journaliers à Saint-Hyacinthe, en 1831 d'après le lieu de résidence (chefs de ménage seulement)

\begin{tabular}{|l|c|c|c|}
\hline \multicolumn{1}{|c|}{ LIEUX } & JOURNALIERS & \multicolumn{2}{|c|}{ JOURNALIERS AGRICOLES } \\
& & Nombre & $\%$ \\
\hline 1. Village de Saint-Hyacinthe & 60 & 0 & 0 \\
2. Saint-Hyacinthe (rangs) & 72 & 54 & 75 \\
3. La Présentation (paroisse) & 40 & 32 & 80 \\
4. Saint-Damase (paroisse) & 36 & 26 & 72 \\
5. Village de Saint-Césaire & 8 & 0 & 0 \\
6. Saint-Césaire (rangs) & 50 & 46 & 92 \\
7. Village de Saint-Pie & 16 & 1 & 6 \\
8. Saint-Pie (rangs) & 39 & 28 & 72 \\
9. Villages (bilan) & 84 & 1 & 1 \\
10. Rangs ou paroisses (bilan) & 237 & 186 & 78 \\
TOTAL & 321 & 187 & 58 \\
\hline
\end{tabular}

Source: Recensement de 1831.

tration de notables ruraux (seigneur, clercs, marchands, médecins, notaires) qui sont, d'une part, des intermédiaires entre l'économie paysanne et l'économie englobante et, d'autre part, des acteurs sociaux qui vivent d'un prélèvement sur la production paysanne ${ }^{17}$.

Cette dernière réflexion nous amène sur le terrain de la structure sociale que les mentions professionnelles n'éclairent guère. Nous nous bornons à quelques constatations sommaires. À part le repérage des notables, normalement concentrés dans les bourgs, et l'identification d'un prolétariat rural, nous pouvons distinguer au sein de la paysannerie, à côté de la masse des propriétaires-exploitants, une faible proportion de fermiers, soit 7,8\% de l'ensemble des exploitants agricoles de la seigneurie en 1831. La proportion est d'environ $9 \%$ dans les secteurs les plus anciens et les plus riches comme La Présentation et NotreDame-du-Rosaire, et moindre dans les paroisses plus récentes. SaintDamase fait toutefois exception avec $12 \%$ de fermiers, mais ces derniers sont concentrés dans des rangs qui longent les plus vieilles paroisses.

Mais pour atteindre la réalité sociale et la différenciation des statuts socio-économiques, il faut faire appel à d'autres sources que les recensements. L'étude de la propriété foncière et des fortunes mobi-

17 Sur la notabilité rurale dans les sociétés paysannes, voir H. Mendras, Sociétés paysannes (Paris, Armand Colin, 1976). 
lières aidera certainement à mieux déterminer la place respective des acteurs dans la hiérarchie sociale des campagnes maskoutaines durant le premier tiers du XIXe siècle.

\section{3 - LA PROPRIÉTÉ FONCIÈRE}

Cette analyse repose à la fois sur le recensement de 1831 et les inventaires après décès. Malgré leurs lacunes respectives ${ }^{18}$, qu'il ne s'agit ni de dissimuler ni de grossir, ces deux sources permettent de saisir l'évolution de la trame foncière dans la seigneurie de Saint-Hyacinthe durant le premier tiers du XIXe siècle, et de déterminer la répartition du sol entre les groupes socio-professionnels et à l'intérieur même de la paysannerie.

Notons d'abord la primauté de la propriété paysanne en 1831 (voir tableau 6). Les cultivateurs accaparent près de $85 \%$ de la superficie totale possédée par les chefs de ménage de la seigneurie. Ces résultats sont similaires à ceux que nous avions obtenus dans une étude antérieure, à partir des inventaires après décès, pour le début du XIXe siècle $^{19}$. Le cas maskoutain n'est d'ailleurs pas unique au Bas-Canada. Nos résultats se comparent à ceux de Fernand Ouellet pour Laprairie et à ceux de Mario Lalancette pour La Malbaie ${ }^{20}$. Certains notables ruraux, en particulier les marchands et les membres des professions libérales du village de Saint-Hyacinthe, possèdent plusieurs terres. Mais cette propriété «bourgeoise» demeure secondaire dans la structure foncière de la seigneurie. La domination de la propriété paysanne apparaît naturellement encore plus évidente dans les paroisses sans bourg comme La Présentation et Saint-Damase.

Les artisans, du moins ceux de la zone rurale de la seigneurie, possèdent de petites terres qui contribuent à leur subsistance. Selon le recensement de 1831, un nombre relativement élevé d'artisans se partage une part plutôt modeste du terroir maskoutain. Quant aux journa-

18 Au recensement de 1831 , la déclaration des biens fonciers par chef de ménage occulte certaines facettes de la réalité dont la présence de plusieurs propriétaires au sein d'un même ménage et la copropriété de biens fonciers par plusieurs individus (voir Mario Lalancette, «Essai sur la répartition de la propriété foncière à La Malbaie, au pays de Charlevoix», F. Lebrun et N. Séguin, op. cit., 63-78). Quant aux inventaires après décès, ils constituent un échantillon des biens fonciers. Or, cet échantillon n'est pas exempt de défauts comme la sous-représentation de certains groupes socio-économiques et de certains groupes d'âge. De plus, il n'est pas toujours possible de vérifier si tous les biens propres des défunts et des conjoints ont été relevés lors des inventaires des biens d'une communauté. La comparaison des données du recensement de 1831 avec celles des inventaires permet d'établir le degré de fiabilité de ces derniers pour l'étude de la propriété foncière.

19 Christian Dessureault, «La propriété rurale et la paysannerie dans la plaine maskoutaine, 1795-1814», F. Lebrun et N. Séguin, op. cit., 39-49.

${ }_{20}$ F. Ouellet, «Répartition de la propriété foncière et types d'exploitation agricole dans la seigneurie de Laprairie durant les années 1830», Éléments d'histoire sociale du Bas-Canada (Montréal, Hurtubise HMH, 1972); M. Lalancette, «Essai sur la répartition de la propriété foncière à La Malbaie, au pays de Charlevoix», 63-78. 


\section{TABLEAU 6}

Ventilation de la propriété foncière maskoutaine par groupe socio-professionnel, 1831 (terres de plus de 1 arpent)

\begin{tabular}{|c|c|c|c|c|c|c|c|c|c|c|c|c|c|c|c|c|c|c|c|c|c|c|c|c|}
\hline \multirow{3}{*}{$\begin{array}{l}\text { Groupes socio- } \\
\text { professionnels }\end{array}$} & \multicolumn{4}{|c|}{ Saint-Hyacinthe } & \multicolumn{4}{|c|}{ La Présentation } & \multicolumn{4}{|c|}{ Saint-Damase } & \multicolumn{4}{|c|}{ Saint-Césaire } & \multicolumn{4}{|c|}{ Saint-Pie } & \multicolumn{4}{|c|}{ Total } \\
\hline & \multicolumn{2}{|c|}{ Propriétaires } & \multicolumn{2}{|c|}{ Superficie } & \multicolumn{2}{|c|}{ Propriétaires } & \multicolumn{2}{|c|}{ Superficie } & \multicolumn{2}{|c|}{ Propriétaires } & \multicolumn{2}{|c|}{ Superficie } & \multicolumn{2}{|c|}{ Propriétaires } & \multicolumn{2}{|c|}{ Superficie } & \multicolumn{2}{|c|}{ Propriétaires } & \multicolumn{2}{|c|}{ Superficie } & \multicolumn{2}{|c|}{ Propriétaires } & \multicolumn{2}{|c|}{ Superficie } \\
\hline & Nombre & $\%$ & Arpents & $\%$ & Nombre & $\%$ & Arpents & $\%$ & Nombre & $\%$ & Arpents & $\%$ & Nombre & $\%$ & Arpents & $\%$ & Nombre & $\%$ & Arpents & $\%$ & Nombre & $\%$ & Arpents & $\%$ \\
\hline 1. Cleres & 1 & 0,2 & 1,247 & 1,7 & - & & - & & 1 & 0,4 & 150 & 0,8 & 1 & & 300 & 0,9 & - & & - & & 3 & 0,2 & 1,697 & 1,0 \\
\hline 2. Professions libérales & 3 & 0,5 & 4,953 & 6,7 & - & & - & & - & & - & & 3 & & 1,429 & 4,6 & 1 & 0,3 & 440 & 1,8 & 1 & 0,4 & 6,822 & 4,0 \\
\hline 3. Marchands & 11 & 2,0 & 4,486 & 6,1 & 2 & 1,0 & 505 & 2,5 & _ & & - & & 2 & & 352 & 1,1 & 2 & 0,7 & $2221 / 2$ & 1,0 & 17 & 1,0 & $5,5651 / 2$ & 3,3 \\
\hline 4. Aubergistes & 4 & 0,7 & 837 & 1,2 & - & & - & & - & & - & & 4 & & 460 & 1,5 & - & & - & & 8 & 0,5 & 1,297 & 0,8 \\
\hline 5. Artisans & 42 & 7,5 & 2,547 & 3,5 & 10 & 4,9 & 482 & 2,4 & 8 & 3,3 & $3401 / 2$ & 1,7 & 20 & & $1,1471 / 2$ & 3,7 & 14 & 4,6 & 1,095 & 4,5 & 94 & 5,5 & $5,6111 \frac{1}{2}$ & 3,3 \\
\hline 6. Cultivateurs & 460 & 82,1 & 56,086 & 76,7 & 181 & 88,7 & $19,2071 / 2$ & 94,2 & 218 & 89,4 & $18,6131 / 2$ & 93,9 & 354 & & $25,9701 / 2$ & 83,3 & 271 & 89,7 & $21,1891 / 2$ & 86,5 & 1,484 & 86,5 & $141,0671 / 2$ & 83,5 \\
\hline 7. Fermiers & 11 & 2,0 & 1,154 & 1,6 & - & & - & & 11 & 4,5 & 588 & 3,0 & 4 & & 320 & 1,0 & 6 & 2,0 & 345 & 1,4 & 32 & 1,9 & 2,407 & 1,4 \\
\hline 8. Joumaliers & 7 & 1,2 & 142 & 0,2 & 8 & 3,9 & 139 & 0,7 & 2 & 0,8 & 61 & 0,3 & 6 & & 242 & 0,8 & 1 & 0,3 & $521 / 2$ & 0,2 & 24 & 1,4 & $6361 / 2$ & 0,4 \\
\hline 9. Veuves & 20 & 3,6 & 1,614 & 2,2 & 2 & 1,0 & 47 & 0,2 & 3 & 1,2 & 64 & 0,3 & 1 & & 60 & 0,2 & 2 & 0,7 & 400 & 1,6 & 28 & 1,6 & 2,185 & 1,3 \\
\hline 10. Autres & 1 & 0,2 & 75 & 0,1 & 1 & 0,5 & 14 & - & 1 & 0,4 & $11 / 2$ & - & 9 & & 904 & 2,9 & 5 & 1,7 & 742 & 3,0 & 17 & 1,0 & $1,7361 / 2$ & 1,0 \\
\hline TOTAL & 560 & 100,0 & 73,141 & 100,0 & 204 & 100,0 & $20,3941 / 2$ & 100,0 & 244 & 100,0 & $19,8181 / 2$ & 100,0 & 404 & & 31,185 & 100,0 & 302 & 100,0 & $24,4861 / 2$ & 100,0 & 1,714 & 100,0 & $169,0251 / 2$ & 100,0 \\
\hline
\end{tabular}


liers, même ceux résidant dans la zone rurale, ce sont habituellement des chefs de ménage sans terre ou propriétaires de toutes petites parcelles.

La superficie moyenne de la propriété paysanne maskoutaine durant le premier tiers du XIXe siècle est remarquablement stable (voir tableau 7). À partir des inventaires après décès, qui fournissent une base de comparaison commune pour l'ensemble de la période, on observe un léger accroissement de cette propriété, soit 113 arpents, entre 1795 et 1804 , et 116 arpents, entre 1825 et 1834 . Cette constatation appuie les historiens qui, en insistant sur la répugnance des familles paysannes à morceler leurs exploitations au-delà du seuil nécessaire à la subsistance, soulignent la résistance de la propriété paysanne et la rigidité des structures foncières dans la société rurale du Québec préindustriel $^{21}$.

TABLEAU 7

Évolution de la propriété paysanne moyenne à Saint-Hyacinthe, 1795-1833

(propriétaires de 1 arpent et plus)

\begin{tabular}{|l|c|}
\hline & Nombre d'arpents \\
\hline $1795-1804$ & 113,4 \\
$1805-1814$ & 115,2 \\
$1825-1834$ & 116,3 \\
Recensement de 1831 & 95,0 \\
\hline
\end{tabular}

Sources: Recensement de 1831, inventaires après décès et registres d'état civil (pour les cas où l'inventaire ne mentionne pas la profession).

D'après le recensement de 1831 , le nombre moyen d'arpents de terre possédés par les chefs de ménage paysans est beaucoup moins élevé que celui obtenu avec les inventaires, mais ceci ne va pas à l'encontre de nos conclusions. Une moyenne de 95 arpents de terre pour la totalité des cultivateurs représente encore un niveau fort respectable. Par ailleurs, si l'utilisation des inventaires après décès conduit à une certaine surévaluation, elle permet tout de même des comparaisons intéressantes dans le temps et dans l'espace.

Les données sur la superficie moyenne des terres montrent une certaine résistance au morcellement dans l'ensemble de la seigneurie, durant le premier tiers du XIXe siècle. Mais, au début des années 1830, la seigneurie de Saint-Hyacinthe combine des terroirs plus anciens et

21 Louis Michel, «Varennes et Verchères, des origines au milieu du XIXe siècle. État d'une enquête», Joseph Goy et Jean-Pierre Wallot, Evolution et éclatement du Monde rural. FranceQuébec XVIIe-XXe siècles (Paris et Montréal, EHESS et PUM, 1986), 325-340. 
d'autres plus récents. On s'attendrait à trouver une moyenne plus élevée dans ces nouveaux secteurs de peuplement, à Saint-Césaire, SaintDamase et Saint-Pie, qui occulterait les résultats de la pression démographique dans les terroirs plus anciens. Mais il n'en est rien. Ce sont au contraire les paysans des terroirs plus anciens de La Présentation et de Notre-Dame-du-Rosaire qui possèdent, en moyenne, la plus grande superficie de terre au tournant des années 1830 , ce que confirment les deux sources utilisées (voir tableau 8).

L'analyse de la répartition de la propriété paysanne par catégorie de superficie, d'après les inventaires après décès, révèle une différenciation de la paysannerie qui s'affirme au cours du premier tiers du XIXe siècle (voir tableau 9). Entre les deux périodes, 1795-1814 et 1825-1834, on observe un accroissement du nombre des petits propriétaires (moins de 90 arpents) et des grands propriétaires (plus de 300 arpents), aux dépens des catégories intermédiaires. Par ailleurs, les données du recensement de 1831 montrent que l'utilisation des inventaires après décès minimise fortement le nombre réel des petits propriétaires, surtout ceux de moins de 60 arpents, et grossit quelque peu celui des grands propriétaires de 300 arpents et plus.

La comparaison de données du recensement de 1831 pour chacune des cinq paroisses maskoutaines montre que la petite propriété, inférieure à 60 arpents, est particulièrement présente dans les secteurs de peuplement récent, à Saint-Césaire, à Saint-Damase et à Saint-Pie où, d'après une interprétation malthusienne, la pression démographique aurait dû intervenir moins fortement sur la trame foncière (voir tableau 10). La grande foncière paysanne est davantage l'apanage des terroirs plus anciens de La Présentation et de Notre-Dame-du-Rosaire, comme si le temps, malgré la pression démographique grandissante, y

\section{TABLEAU 8}

Propriété foncière moyenne des cultivateurs maskoutains, 1825-1834 (propriétaires de 1 arpent et plus)

\begin{tabular}{|l|c|c|}
\hline & $\begin{array}{c}\text { Recensement de } \\
1831\end{array}$ & $\begin{array}{c}\text { Inventaires après décès } \\
1825-1834\end{array}$ \\
\hline & Nombre d'arpents & Nombre d'arpents \\
\hline Saint-Hyacinthe & 121,9 & 153,2 \\
La Présentation & 106,1 & 113,8 \\
Saint-Damase & 85,4 & 86,9 \\
Saint-Césaire & 73,4 & 84,1 \\
Saint-Pie & 78,2 & 68,1 \\
\hline
\end{tabular}

Sources: Voir tableau 7. 
TABLEAU 9

Répartition de la propriété foncière paysanne dans la seigneurie de Saint-Hyacinthe, 1795-1834 (pour les propriétaires de plus de 1 arpent de terre)

\begin{tabular}{|c|c|c|c|c|c|c|c|c|c|c|c|c|}
\hline \multirow[t]{2}{*}{ Années } & & \multicolumn{10}{|c|}{ Catégorie de propriétaires selon le nombre d'arpents de terre possédés } & \multirow[t]{2}{*}{ Total } \\
\hline & & $1-9$ & $10-29$ & $30-59$ & $60-89$ & $90-119$ & $120-179$ & $180-239$ & $240-299$ & $300-419$ & + de 420 & \\
\hline \multirow{2}{*}{$1795-1814$} & Nombre & & & 18 & 59 & 69 & 35 & 20 & 10 & 5 & 2 & 218 \\
\hline & $\%$ & & & 8,3 & 27,1 & 31,5 & 16,1 & 9,2 & 4,6 & 2,3 & 0,9 & 100,0 \\
\hline \multirow{2}{*}{$1825-1834$} & Nombre & & 3 & 27 & 75 & 43 & 32 & 10 & 5 & 7 & 8 & 210 \\
\hline & $\%$ & & 1,4 & 12,9 & 35,7 & 20,5 & 15,2 & 4,8 & 2,4 & 3,3 & 3,8 & 100 \\
\hline \multirow{2}{*}{1831} & Nombre & 14 & 45 & 260 & 569 & 245 & 188 & 93 & 34 & 23 & 13 & 1484 \\
\hline & $\%$ & 0,9 & 3,9 & 17,5 & 38,3 & 16,5 & 12,7 & 6,3 & 2,3 & 1,6 & 0,9 & 100 \\
\hline
\end{tabular}

Sources: Recensement de 1831, inventaires après décès et registres d'état civil. 
TABLEAU 10

Répartition de la propriété foncière paysanne dans les paroisses maskoutaines d'après le recensement de $\mathbf{1 8 3 1}$ (cultivateurs propriétaires de 1 arpent et plus)

\begin{tabular}{|c|c|c|c|c|c|c|c|c|c|c|c|}
\hline & \multicolumn{10}{|c|}{ Catégorie selon le nombre d'arpents possédés } & \multirow[b]{2}{*}{ Total } \\
\hline & $1-9$ & $10-29$ & $30-59$ & $60-89$ & $90-119$ & $120-179$ & $180-239$ & $240-299$ & $300-419$ & + de 420 & \\
\hline \multicolumn{12}{|l|}{ SAINT-HYACINTHE } \\
\hline $\mathrm{Nb}$ de propriétaires & 5 & 9 & 42 & 142 & 86 & 83 & 52 & 17 & 14 & 10 & 460 \\
\hline$\%$ & 1,1 & 2,0 & 9,1 & 30,9 & 18,7 & 18,0 & 11,3 & 3,7 & 3,0 & 2,2 & 100,0 \\
\hline \multicolumn{12}{|l|}{ LA PRÉSENTATION } \\
\hline $\mathrm{Nb}$ de propriétaires & 3 & 3 & 23 & 55 & 39 & 31 & 13 & 10 & 3 & 1 & 181 \\
\hline$\%$ & 1,7 & 1,7 & 12,7 & 30,4 & 21,5 & 17,1 & 7,2 & 5,5 & 1,7 & 0,5 & 100,0 \\
\hline \multicolumn{12}{|l|}{ SAINT-DAMASE } \\
\hline $\mathrm{Nb}$ de propriétaires & 1 & 10 & 38 & 96 & 32 & 24 & 12 & 3 & 2 & - & 218 \\
\hline$\%$ & 0,5 & 4,6 & 17,4 & 44,0 & 14,7 & 11,0 & 5,5 & 1,4 & 0,9 & & 100,0 \\
\hline \multicolumn{12}{|l|}{ SAINT-CÉSAIRE } \\
\hline $\mathrm{Nb}$ de propriétaires & 3 & 21 & 91 & 151 & 54 & 23 & 7 & 1 & 1 & 2 & 354 \\
\hline$\%$ & 0,8 & 5,9 & 25,7 & 42,7 & 15,2 & 6,5 & 2,0 & 0,3 & 0,3 & 0,6 & 100,0 \\
\hline \multicolumn{12}{|l|}{ SAINT-PIE } \\
\hline $\mathrm{Nb}$ de propriétaires & 2 & 2 & 66 & 125 & 34 & 27 & 9 & 3 & 3 & - & 271 \\
\hline$\%$ & 0,7 & 0,7 & 24,4 & 46,1 & 12,6 & 10,0 & 3,3 & 1,1 & 1,1 & & 100,0 \\
\hline
\end{tabular}

Source: Recensement de 1831 . 
avait favorisé l'accumulation et la consolidation des avoirs fonciers. Globalement, le glissement vers la petite propriété paysanne est assez net lorsqu'on passe des anciens aux nouveaux terroirs.

Comme facteurs explicatifs de cette dynamique foncière, il ne convient certainement pas d'invoquer le vieil argument des pratiques successorales égalitaires qui entraînent inévitablement le morcellement des terres. Néanmoins, la pression démographique, la rareté réelle ou spéculative des terres neuves et l'accroissement des charges seigneuriales ont certainement influé sur l'évolution de la propriété dans les nouveaux terroirs. Des recherches subséquentes permettront d'évaluer le poids respectif de ces différents facteurs.

Par ailleurs, la consolidation des patrimoines fonciers dans les paroisses plus anciennes semble liée à un mouvement de différenciation sociale au sein de la paysannerie. La concentration de la propriété au profit d'un nombre plus réduit de familles s'accompagne d'une complexité croissante de la structure des avoirs fonciers, qui comptent de plus en plus souvent, comme le montrent les inventaires après décès, plusieurs terres ou plusieurs parcelles dispersées. Cette transformation de l'espace rural, qui s'effectue au gré de deux mouvements apparemment contradictoires, consolidation et parcellisation, est l'expression d'un jeu économique et social où certaines familles paysannes, plus solidement implantées, récupèrent patiemment les terres que d'autres familles ont dû abandonner. En outre, la pression démographique gonfle graduellement le nombre de journaliers résidant dans les campagnes et, surtout, multiplie le nombre de migrants, au fur et à mesure que les places se raréfient. Mais, si nous observons les nouveaux secteurs de peuplement où les colons doivent généralement se satisfaire de moins de terre, il semble assez évident que ce redéploiement dans l'espace n'est pas neutre, ni économiquement ni socialement. Au tournant des années 1830, cette sélectivité socio-économique de la population migrante apparaît d'autant plus criante que les nouvelles paroisses offrent des conditions d'établissement de moins en moins attrayantes ${ }^{22}$.

\section{4 - NIVEAUX DE FORTUNE MOBILIÈRE ET GROUPES SOCIO-PROFESSIONNELS}

L'une des interprétations majeures de l'historiographie sur la société rurale bas-canadienne durant le premier tiers du XIXe siècle est celle de l'appauvrissement global de la paysannerie et, par voie de conséquence, des artisans et des notables ruraux qui vivent indirectement du fruit du travail de la terre. À partir des inventaires après décès, nous

22 Sur l'accroissement des charges seigneuriales dans les nouveaux terroirs de peuplement au début du XIXe siècle, voir S. Courville, «La crise agricole au Bas-Canada. Éléments d'une réflexion géographique (deuxième partie)», Cahiers de géographie du Québec, 24,63 (1980), $385-428$ 
pouvons saisir l'évolution des niveaux de fortune mobilière de la population maskoutaine au cours de cette période et examiner la distribution dans l'espace des fortunes paysannes. Nous ne reprenons pas ici la critique des inventaires après décès, mais présentons simplement une série de données brutes sur la composition et les niveaux de fortune mobilière que des analyses subséquentes plus détaillées avaliseront ou infirmeront.

L'étude des niveaux de fortune de la notabilité rurale exige, à coup sûr, un élargissement du terrain d'enquête. Néanmoins, à partir de ces résultats fragmentaires, il apparaît que l'échelle des fortunes mobilières respecte, voire consacre la hiérarchie traditionnelle du monde rural (voir tableaux 11a et 11b). Au sommet de la hiérarchie des fortunes, le seigneur ${ }^{23}$ et le curé ${ }^{24}$ dont la richesse repose d'abord sur la perception de rentes sur la terre et sur ses fruits. Quelques gros marchands ruraux, actifs dans le commerce de détail comme dans le commerce des grains, intermédiaires privilégiés entre l'économie locale et l'économie globale, accumulent également des actifs mobiliers considérables comparativement à ceux de l'ensemble de la population. Toutefois, la fortune marchande, intrinsèquement liée aux activités commerciales, comprend habituellement une forte proportion de créances, quelquefois difficiles à percevoir, et elle est habituellement grevée de dettes assez lourdes envers les fournisseurs, qui contrebalancent en bonne partie la valeur des créances. Malgré cela, la richesse collective des marchands apparaît plus imposante que celle du clergé et des seigneurs dans la plaine maskoutaine du premier tiers du XIXe siècle. Le commerce rural recrute un assez grand nombre d'agents tantôt en relation, tantôt en concurrence, répartis aux divers étages de la fortune et du pouvoir. À un niveau intermédiaire de la hiérarchie des fortunes, vraisemblablement celui de la petite élite locale, active dans les institutions paroissiales et dans la direction subalterne des milices, on trouve un ensemble hétéroclite de médecins, notaires, petits marchands, artisans et paysans aisés. Il serait intéressant de connaître leurs liens familiaux et les relations économiques qu'ils entretiennent les uns vis-à-vis des autres. Durant le premier tiers du XIXe siècle, la notabilité rurale maskoutaine connaît une phase rapide d'expansion. La forte croissance démographique et l'essor villageois alimentent le développement des activités commerciales et contribuent à asseoir les fortunes marchandes.

23 L'inventaire après décès de Jean Dessaulles, seigneur de Saint-Hyacinthe, du 15 septembre 1835, est malheureusement disparu des minutes du notaire A.-A. Papineau. Néanmoins, le partage de sa succession, du 14 mai 1852 , dresse un bilan rétrospectif de la fortune mobilière des Dessaulles lors de l'inventaire. Les actifs mobiliers auraient alors totalisé près de 250000 livres ancien cours, dont plus de 220000 livres pour les créances. Par ailleurs, les dettes de la succession se montaient alors à 113000 livres ancien cours. ANQM, minutes D.-E. Papineau, no 2982 , le 14 mai 1852 .

${ }_{24}$ L'inventaire après décès de A. Girouard, curé de Notre-Dame-du-Rosaire, du 27 août 1832, est également disparu des minutes du notaire D.-G. Morisson. Toutefois, nous disposons d'une transcription du document original faite par J.-A. Dubreuil, procureur du Séminaire de Saint-Hyacinthe, le 24 mars 1910. ASHSH, section A, série A, dossier 1.1. 
TABLEAU 11a

Fortune mobilière et groupes socio-professionnels dans la seigneurie de Saint-Hyacinthe, 1795-1814

\begin{tabular}{|c|c|c|c|c|c|c|c|c|c|c|c|}
\hline $\begin{array}{c}\text { Groupes } \\
\text { socio-professionnels }\end{array}$ & $\begin{array}{c}\text { Nombre } \\
\text { d'inventaires }\end{array}$ & $\begin{array}{l}\text { Biens de con- } \\
\text { sommation } \\
\text { (en livres) }\end{array}$ & $\begin{array}{l}\text { Biens de } \\
\text { production } \\
\text { (en livres) }\end{array}$ & $\begin{array}{l}\text { Cheptel } \\
\text { (en livres) }\end{array}$ & $\begin{array}{c}\text { Stock } \\
\text { (en livres) }\end{array}$ & $\begin{array}{l}\text { Numéraire } \\
\text { (en livres) }\end{array}$ & $\begin{array}{l}\text { Argenterie } \\
\text { (en livres) }\end{array}$ & $\begin{array}{l}\text { Créances } \\
\text { (en livres) }\end{array}$ & $\begin{array}{l}\text { Actifs totaux } \\
\text { (en livres) }\end{array}$ & $\begin{array}{c}\text { Passifs } \\
\text { (en livres) }\end{array}$ & $\begin{array}{c}\text { Passifs } \\
\text { Actifs } \\
\%\end{array}$ \\
\hline Curé & 1 & 3,162 & 549 & 576 & 672 & 190 & - & 7,830 & 13,429 & 6,353 & 47 \\
\hline Marchands & 2 & 881 & 282 & 1,159 & 1,705 & - & 18 & 4,561 & 8,606 & 14,783 & 172 \\
\hline Professions libérales & 2 & 206 & 98 & 137 & 7 & - & - & - & 448 & 1,758 & 392 \\
\hline Aubergiste & 1 & 645 & 102 & 69 & 539 & - & - & 5,663 & 7,018 & 17,665 & 252 \\
\hline Artisans & 15 & 322 & 134 & 216 & 182 & 1 & - & 351 & 1,206 & 1,722 & 143 \\
\hline Cultivateurs & 248 & 204 & 104 & 348 & 330 & 37 & - & 203 & 1,226 & 737 & 60 \\
\hline Journaliers & 8 & 54 & 37 & 34 & 43 & 25 & - & 35 & 228 & 270 & 118 \\
\hline Veuves & 6 & 722 & 99 & 287 & 86 & 1,053 & 721 & 134 & 3,102 & 1,244 & 40 \\
\hline Autres & 1 & 118 & 67 & 216 & - & 8 & - & - & 409 & 3,724 & 911 \\
\hline
\end{tabular}

Sources: Inventaires après décès et registres d'état civil.

TABLEAU 11b

Fortune mobilière et groupes socio-professionnels dans la seigneurie de Saint-Hyacinthe, 1825-1834

\begin{tabular}{|c|c|c|c|c|c|c|c|c|c|c|c|}
\hline $\begin{array}{c}\text { Groupes } \\
\text { socio-professionnels }\end{array}$ & $\begin{array}{c}\text { Nombre } \\
\text { d'inventaires }\end{array}$ & $\begin{array}{c}\text { Biens de con- } \\
\text { sommation } \\
\text { (en livres) }\end{array}$ & $\begin{array}{l}\text { Biens de } \\
\text { production } \\
\text { (en livres) }\end{array}$ & $\begin{array}{l}\text { Cheptel } \\
\text { (en livres) }\end{array}$ & $\begin{array}{c}\text { Stocks } \\
\text { (en livres) }\end{array}$ & $\begin{array}{l}\text { Numéraire } \\
\text { (en livres) }\end{array}$ & $\begin{array}{l}\text { Argenterie } \\
\text { (en livres) }\end{array}$ & $\begin{array}{l}\text { Créances } \\
\text { (en livres) }\end{array}$ & $\begin{array}{l}\text { Actifs totaux } \\
\text { (en livres) }\end{array}$ & $\begin{array}{c}\text { Passifs } \\
\text { (en livres) }\end{array}$ & $\begin{array}{c}\text { Passifs/ } \\
\text { Actifs } \\
\%\end{array}$ \\
\hline Curé & 1 & 12,160 & 1,027 & 11,250 & 1,721 & 1,012 & * & 19,460 & 46,630 & 14,045 & 30 \\
\hline Marchands & 5 & 1,550 & 373 & 1,044 & 2,099 & 44 & 95 & 30,680 & 35,680 & 24,376 & 68 \\
\hline Professions libérales & 1 & 1,305 & 147 & 694 & 180 & - & 181 & 422 & 2,929 & 3,429 & 117 \\
\hline Aubergiste & 1 & 181 & 81 & 162 & 202 & 36 & - & - & 662 & 1,770 & 267 \\
\hline Huissier & 1 & 334 & 52 & 252 & 36 & - & - & 98 & 772 & 3,638 & 471 \\
\hline Artisans & 10 & 282 & 138 & 230 & 295 & 51 & - & 435 & 1,431 & 1,683 & 118 \\
\hline Cultivateurs & 235 & 210 & 122 & 444 & 346 & 22 & - & 341 & 1,485 & 1,182 & 80 \\
\hline Journaliers & 21 & 78 & 44 & 77 & 11 & 17 & - & 38 & 265 & 197 & 74 \\
\hline Autres & 5 & 46 & 6 & 63 & 19 & 8 & - & 447 & 589 & 397 & 67 \\
\hline
\end{tabular}

* Il y avait de l'argenterie dans l'inventaire après décès du curé A. Girouard, mais elle n'a pas été évaluée.

Sources: Inventaires après décès et registres d'état civil. 
Les actifs mobiliers des artisans maskoutains sont à peu près comparable à ceux des cultivateurs. Toutefois, la valeur de leurs biens de consommation, qui constitue un meilleur indice du niveau de vie, montre des conditions d'existence matérielle généralement plus favorables. Mais celles-ci varient beaucoup d'un inventaire à l'autre. Certains artisans, comme les cordonniers et les menuisiers, se retrouvent plus souvent au bas de l'échelle tandis que d'autres, comme les boulangers et les ferblantiers, accumulent des fortunes comparables à celles des petits marchands. L'investissement en biens de production demeure fort réduit. En somme, l'industrie rurale est le fait de toutes petites entreprises artisanales.

Comme on pouvait s'y attendre, les journaliers se situent globalement au bas de l'échelle des fortunes mobilières. La moyenne de leurs actifs mobiliers, autour de 250 livres, est largement inférieure à celle des autres groupes. Par ailleurs, le corpus des inventaires après décès de journaliers se caractérise par son homogénéité. Un seul des 29 inventaires après décès enregistre des actifs mobiliers supérieurs à 500 livres $^{25}$. La faible valeur des biens de consommation reflète la précarité des conditions de travail. Malgré la pauvreté des journaliers, la présence de numéraire et de créances dans leurs inventaires est un indice précieux sur leur type d'insertion dans le marché local de la main-d'oeuvre. Par ailleurs, les journaliers ruraux possèdent habituellement plusieurs outils d'agriculture utilisés dans le potager ou le lopin de pommes de terre, ainsi que quelques animaux, normalement un ou deux porcs. La multiplication des inventaires de journaliers dans la seconde période illustre bien l'accroissement du prolétariat rural qui représente, au recensement de 1831 , plus de $13 \%$ des chefs de ménage de la seigneurie de SaintHyacinthe. Cette augmentation du nombre de journaliers entraîne assurément une hausse proportionnelle des fortunes mobilières de niveau médiocre dans l'ensemble de la communauté rurale. Des recherches subséquentes, à partir des registres d'état civil, devront s'attaquer plus directement aux modalités de formation et de reproduction de ce prolétariat rural.

Quant aux cultivateurs maskoutains, le nombre considérable d'inventaires après décès permet non seulement de fixer les grands traits de leur fortune mobilière et de suivre son évolution, mais aussi de procéder à une ventilation plus fine de ces avoirs pour saisir la stratification interne du groupe. Au cours du premier tiers du XIXe siècle, les actifs mobiliers moyens des paysans de connaissent une assez forte croissance, passant de 1296 livres entre 1795 et 1814 à plus de 1485 livres entre 1825 et 1834 . La hausse réelle des actifs est certainement encore plus élevée, compte tenu de la baisse générale des prix au cours de la

25 En 1832, les actifs mobiliers de J. Laforme, journalier à Notre-Dame-du-Rosaire, totalisaient 590 livres. ANQM, minutes D.-G. Morisson, no 159, le 3 octobre 1832. 
période. En somme, il n'y a pas d'appauvrissement global de la paysannerie maskoutaine durant cette période, mais plutốt une consolidation des avoirs fonciers et mobiliers, voire un enrichissement.

Il y a d'ailleurs une concordance dans l'évolution de plusieurs composantes de la fortune paysanne. En premier lieu, notons la stabilité des biens de consommation dans les inventaires après décès. L'analyse de cette catégorie permettrait de mieux mesurer l'évolution réelle des niveaux de vie. Nous signalons toutefois, à titre qualitatif, certains changements intervenus au cours de la période, comme la généralisation du poêle de fer et la diffusion des horloges dans les couches moyennes et supérieures de la paysannerie ${ }^{26}$.

En second lieu, la croissance importante de la valeur des biens de production et du cheptel témoigne du développement de l'agriculture maskoutaine durant le premier tiers du XIXe siècle. L'investissement dans l'équipement agricole accapare toujours une part relativement faible du capital de production et nous n'avons pas observé de transformations radicales de ce matériel qui demeure rudimentaire. Parmi les améliorations plus facilement perceptibles, notons la diffusion de la herse à dents de fer qui s'ajoute à l'outil traditionnel à dents de bois, dans la majorité des inventaires, ainsi qu'une augmentation globale du nombre d'outils. Les progrès sont beaucoup plus évidents dans le secteur du textile domestique avec un accroissement considérable des rouets et, surtout, des métiers à tisser.

La forte augmentation de la valeur du cheptel est plus difficile à interpréter. Certes, ces gains importants dans le secteur de l'élevage infirment la perspective d'une crise globale de l'agriculture. Mais il faudra pousser l'analyse plus à fond pour savoir s'il s'agit simplement d'une consolidation de l'exploitation paysanne traditionnelle, où le bétail sert d'abord de support et de complément à la production céréalière, ou si nous sommes en face d'une percée embryonnaire de l'élevage proprement dit en tant que spécialisation. Globalement, l'observation des données brutes des inventaires après décès appuie davantage la première hypothèse.

Par ailleurs, l'accroissement des créances entre les deux périodes est largement contrebalancée par la hausse considérable des dettes passives dont la proportion par rapport aux actifs mobiliers passent de 60 à $80 \%$. Cette augmentation des créances, et surtout des dettes passives, suggère une intensification des réseaux de crédit dans les campagnes et une pénétration croissante de l'économie de marché. Il conviendra toutefois d'identifier l'origine de cet endettement paysan, d'évaluer le poids des dettes marchandes et seigneuriales dans le bilan des fortunes, de

26 Les poêles de fer et les horloges sont des objets de consommation assez coûteux. Leur prix varie de 60 à 120 livres. 
lier l'évolution de la dette à la conjoncture économique et, dans la mesure du possible, à la valeur des terres, une donnée fondamentale qui fait défaut ${ }^{27}$.

\section{5 - NIVEAUX DE FORTUNE MOBILIÈRE ET DIFFÉRENCIATION SOCIALE AU SEIN DE LA PAYSANNERIE}

À l'instar de la propriété foncière, la fortune mobilière paysanne est très inégalement répartie entre les cinq paroisses de la seigneurie de Saint-Hyacinthe vers 1830 (voir tableau 12). Globalement, les fortunes des paysans des terroirs plus anciens sont beaucoup plus élevées que celles des paroisses de peuplement récent. Par rapport au début du XIXe siècle, la fortune mobilière des paysans de Notre-Dame-du-Rosaire et de La Présentation s'est considérablement accrue. Par contre, celle des paysans de Saint-Césaire et de Saint-Pie demeure bien en deçà du niveau atteint par l'ensemble de la paysannerie maskoutaine du début du siècle. Les données sur diverses composantes confirment la répartition générale. Au début des années 1830 , les nouveaux secteurs de peuplement de Saint-Césaire et de Saint-Pie apparaissent comme des ilôts de pauvreté, voire de misère. La valeur des biens de consommation suggère un niveau de vie médiocre tandis que la valeur des biens de production et du cheptel révèle la faiblesse des exploitations et les limites à leur développement. Enfin, comme dernière ombre à un tableau plutôt sombre, le taux d'endettement de ces paysans est bien supérieur à celui des paysans des vieilles paroisses de la seigneurie.

Mais l'ancienneté d'un terroir n'est pas seule en cause. Par exemple, les fortunes des paysans de Saint-Damase font presque le double de celles de Saint-Césaire, bien que ces deux paroisses aient été délimitées récemment, à peu près à la même date. Les données des inventaires sont d'ailleurs confirmées par les rapports du clergé selon lesquels Saint-Césaire ne pouvait pas assurer la subsistance d'un curé en 1823 et encore difficilement en 1829, alors que Saint-Damase fournissait dès le début un revenu curial suffisant et fort confortable en $1829^{28}$.

27 La propriété foncière représente la principale composante de la fortune paysanne. Il y a d'ailleurs des rapports entre le niveau de fortune mobilière et la superficie de la terre. Les coefficients de corrélation entre ces deux éléments sont respectivement de .6113 pour la période 1795 1814 et de .6108 pour les années $1825-1834$.

28 ASHSH, série A, 1 a, dossier 4. Délimitation de deux nouvelles paroisses à SaintHyacinthe, le 9 novembre 1817; AESH, dossier 18, Lettre de J. Poirier, curé de Saint-Césaire à J.-P. Plessis, évêque de Québec, le 24 février 1823. En 1829, les habitants de Saint-Damase, dont la paroisse comptait alors 1822 âmes, «déclarent fournir annuellement par leurs dixmes pour la subsistance d'un prêtre qui leur est donné la quantité de 800 minots de blé froment, 300 minots de pois, 400 minots d'avoine, 100 minots d'autres grains...» AESH, dossier 23, Requête des habitants de la seigneurie Debartzch pour l'érection canonique de la paroisse de Saint-Damase, 17 et 29 mai 1829. Les habitants de Saint-Césaire dont la paroisse comptait environ 2000 âmes en 1831, ne peuvent «fournir annuellement par leurs dixmes pour la subsistance d'un prêtre la quantité de 300 minots de blé froment, 100 minots de pois, 200 minots d'avoine, 25 minots de blé d'inde et 25 minots d'orge». AESH, dossier 1B, Requête pour l'érection canonique de la paroisse de Saint-Césaire, le 10 août 1831. 
TABLEAU 12

Les fortunes paysannes dans la plaine maskoutaine, par paroisse, 1825-1834

\begin{tabular}{|c|c|c|c|c|c|c|c|c|c|c|}
\hline PAROISSES & $\begin{array}{c}\text { Nombre } \\
\text { d'inventaires }\end{array}$ & $\begin{array}{l}\text { Biens de con- } \\
\text { sommation } \\
\text { (en livres) }\end{array}$ & $\begin{array}{l}\text { Biens de } \\
\text { production } \\
\text { (en livres) }\end{array}$ & $\begin{array}{l}\text { Cheptel } \\
\text { (en livres) }\end{array}$ & $\begin{array}{c}\text { Stocks } \\
\text { (en livres) }\end{array}$ & $\begin{array}{l}\text { Numéraire } \\
\text { (en livres) }\end{array}$ & $\begin{array}{l}\text { Créances } \\
\text { (en livres) }\end{array}$ & $\begin{array}{l}\text { Actifs totaux } \\
\text { (en livres) }\end{array}$ & $\begin{array}{l}\text { Passifs } \\
\text { (en livres) }\end{array}$ & $\begin{array}{c}\text { Passifs/ } \\
\text { Actifs } \\
\%\end{array}$ \\
\hline LA PRÉSENTATION & 48 & 235 & 144 & 475 & 501 & 28 & 271 & 1,654 & 1,195 & 72 \\
\hline SAINT-HYACINTHE & 93 & 239 & 134 & 526 & 350 & 24 & 526 & 1,799 & 1,485 & 83 \\
\hline SANT-DAMASE & 46 & 199 & 120 & 468 & 414 & 31 & 210 & 1,442 & 910 & 63 \\
\hline SAINT-CÉSAIRE & 36 & 141 & 79 & 224 & 122 & 3 & 168 & 737 & 761 & 103 \\
\hline SAINT-PIE & 12 & 147 & 87 & 277 & 115 & - & 258 & 884 & 1,170 & 132 \\
\hline MOYENNE & 235 & 210 & 122 & 444 & 346 & 22 & 341 & 1,485 & 1,182 & 80 \\
\hline
\end{tabular}

Sources: Inventaires après décès et registres d'état civil. 
On retrouve le même genre de contraste dans des paroisses plus anciennes comme Notre-Dame-du-Rosaire et La Présentation. Ainsi, entre 1795 et 1814 , la moyenne des fortunes mobilières de 29 habitants du Grand Rang est de 2677 livres alors que celle des 22 ménages inventoriés à la rivière Salvail n'est que de 1394 livres et cette inégalité persiste encore entre 1825 et $1834^{29}$. En 1806 , le curé Girouard résume la situation: «Ce sont, écrit-il à l'évêque, les meilleures gerbes de mon champ, les meilleures [herbes] de mes pâturages et les brebis les plus grasses qui sont dans les équerres des Soixante et du Grand Rang et dans le haut des Etangs. Salvail depuis longtemps habité n'a jamais valu (les comptes de dîmes en font foi) la moitié de ces endroits.» ${ }^{30}$

Pour expliquer ces regroupements de paysans pauvres et de paysans bien nantis, il faudrait examiner de plus près la qualité des sols, le rythme du mouvement de colonisation et, surtout, les origines géographiques et sociales des colons. Mais ces exemples prouvent amplement l'existence d'une hiérarchie au sein de la paysannerie. La ventilation des fortunes mobilières dans l'ensemble de la seigneurie et dans chacune des cinq paroisses permet de cerner les inégalités. Nous nous sommes déjà penché sur ce phénomène dans un article précédent mais cette étude s'arrêtait en $1814^{31}$. Les 235 inventaires recueillis - pour la période 1825-1834 - prolongent notre recherche initiale et confirment ses résultats.

Au début du XIXe siècle, plus du tiers des inventaires paysans enregistrent des actifs mobiliers inférieurs à 500 livres (voir tableau 13). Le niveau de fortune mobilière de ces familles paysannes est comparable à celui des journaliers ruraux. À l'instar de ces derniers, ils vivent dans des conditions matérielles relativement médiocres. Ils doivent assurément travailler à l'extérieur de leur propre exploitation, du moins de manière complémentaire, pour assurer la subsistance de leur famille. Au tournant des années 1830 , la proportion des actifs mobiliers inférieurs à 500 livres n'a pas beaucoup diminué. Ils représentent toujours près du tiers des inventaires paysans.

Entre 501 et 1000 livres d'actifs mobiliers, ce n'est certainement pas l'aisance matérielle, mais ce n'est ni l'indigence ni la pauvreté des journaliers et de la couche inférieure de la paysannerie. À ce niveau de fortune, la famille paysanne approche le seuil de l'autosubsistance, mais elle ne jouit pas pour autant d'une véritable autonomie économique.

\footnotetext{
29 Soit une moyenne de 1124 livres pour 14 inventaires paysans de Salvail et 2424 livres pour 15 inventaires de la partie orientale du Grand Rang.

30 AESH, dossier 67, Lettre de A. Girouard, curé de Notre-Dame-du-Rosaire à J.-O. Plessis, évêque de Québec, le 12 avril 1806; voir aussi la lettre de P. Bardy, curé de La Présentation à J.-O. Plessis, évêque de Québec, le 16 octobre 1811, AESH, dossier 68 .

31 C. Dessureault, «L'égalitarisme paysan dans l'ancienne société rurale de la vallée du Saint-Laurent: éléments pour une ré-interprétation», $R H A F, 40,3$ (hiver 1987): 373-407.
} 
TABLEAU 13

Répartition des fortunes mobilières paysannes, 1795-1834

\begin{tabular}{|c|c|c|c|c|}
\hline \multirow{3}{*}{$\begin{array}{c}\text { Niveaux des } \\
\text { actifs mobiliers } \\
\text { (en livres ancien cours) }\end{array}$} & \multicolumn{4}{|c|}{ Années } \\
\hline & \multicolumn{2}{|c|}{$1795-1814$} & \multicolumn{2}{|c|}{ 1825-1834 } \\
\hline & Nombre & $\%$ & Nombre & $\%$ \\
\hline Carence d'inventaire & 6 & 2,4 & 5 & 2,1 \\
\hline Moins de 500 livres & 86 & 34,7 & 74 & 31,5 \\
\hline $501-1000$ livres & 69 & 27,8 & 51 & 21,7 \\
\hline $1001-2000$ livres & 53 & 21,4 & 56 & 23,8 \\
\hline $2001-4000$ livres & 22 & 8,9 & 36 & 15,4 \\
\hline $4001-8000$ livres & 10 & 4,0 & 8 & 3,4 \\
\hline Plus de 8000 livres & 2 & 0,8 & 5 & 2,1 \\
\hline Total & 248 & 100,0 & 235 & 100,0 \\
\hline
\end{tabular}

Sources: Inventaires après décès et registres d'état civil.

Elle demeure fortement sensible aux fluctuations économiques et aux accidents de toute sorte qui ponctuent la vie rurale. Elle ressent durement les prélèvements extérieurs sur la production agricole. Au début du siècle, plus de $28 \%$ des inventaires paysans se situent dans cette catégorie. Entre 1825 et 1834 , cette proportion chute à environ $22 \%$.

Cette diminution des actifs mobiliers de 501 à 1000 livres a profité partiellement à la frange intermédiaire de la paysannerie, celle qui possède de 1001 à 2000 livres d'actifs mobiliers, dont le pourcentage s'accroît de 22 à $24 \%$ des inventaires paysans. Ces familles paysannes ont davantage accès au confort matériel et à l'univers de la consommation des produits exotiques, grâce à une production agricole excédentaire. En fixant autour de 1000 livres d'actifs mobiliers le seuil plutôt relatif de l'autonomie économique et de l'aisance matérielle, c'est encore plus de la moitié des inventaires paysans qui, entre 1825 et 1834 , ne peuvent participer avantageusement à l'économie de marché.

La forte croissance de la moyenne des actifs mobiliers des paysans maskoutains a d'abord contribué à consolider les fortunes de la couche supérieure de la paysannerie, entre 2001 et 4000 livres d'actifs mobiliers, dont le pourcentage passe de 10 à $15 \%$ des inventaires paysans entre 1795-1814 et 1825-1834. Par ailleurs, la proportion des paysans plus riches, dont le niveau des actifs mobiliers, de 4000 livres et plus, se compare à celui d'une partie des notables ruraux, demeure stationnaire autour de $5 \%$ des inventaires paysans.

Mais cette hiérarchie des fortunes paysannes n'est pas tout à fait la même dans les cinq paroisses de la seigneurie (voir tableau 14). Dans chacune, les clivages au sein de la paysannerie demeurent partout pré- 
sents. Cependant, la richesse constitue d'abord le privilège des terroirs de peuplement plus ancien tandis que la pauvreté est davantage présente dans les terroirs de peuplement récent. A Saint-Césaire, la distribution des richesses mobilières paysannes apparaît même dramatiquement tronquée vers les échelons inférieurs de la richesse. Plus des trois quarts des inventaires paysans enregistrent des actifs mobiliers inférieurs à 1000 livres.

TABLEAU 14

Répartition des fortunes mobilières paysannes dans les paroisses maskoutaines, 1825-1834

\begin{tabular}{|l|cc|cc|}
\hline Nom de la paroisse & \multicolumn{2}{|c|}{ Moins de 1000 livres } & \multicolumn{2}{|c|}{ Plus de 1000 livres } \\
\hline & Nombre & $\%$ & Nombre & $\%$ \\
\cline { 2 - 5 } La Présentation & 25 & 52 & 23 & 48 \\
Saint-Hyacinthe & 48 & 52 & 44 & 48 \\
Saint-Damase & 23 & 50 & 23 & 50 \\
Saint-Césaire & 28 & 78 & 8 & 22 \\
Saint-Pie & 7 & 58 & 5 & 42 \\
\hline
\end{tabular}

Sources: Inventaires après décès et registres d'état civil.

\section{CONCLUSION}

Société rurale d'Ancien Régime en plein marasme économique ou campagnes résolument engagées sur la voie de la modernisation? La seigneurie de Saint-Hyacinthe du premier tiers du XIXe siècle ne correspond ni à l'un ni à l'autre de ces deux modèles globalisants. Les résultats de l'enquête sur les structures professionnelles en 1831, sur l'évolution de la propriété foncière et sur celle de la richesse mobilière renvoient à une situation beaucoup plus nuancée et diversifiée.

Au début du siècle, la seigneurie de Saint-Hyacinthe est une région de colonisation. Dans les deux décennies qui suivent, la croissance démographique demeure particulièrement forte et, jusqu'en 1825 , la seigneurie accepte les surplus démographiques des autres paroisses de la vallée du Saint-Laurent. Toutefois, à partir de 1825, cette croissance démographique s'essouffle et, vers 1830, on perçoit les premiers symptômes d'un blocage agraire.

Notre analyse de la structure professionnelle en 1831 a démontré que les Maskoutains ne sont pas engagés dans un mouvement de protoindustrialisation des campagnes et que l'agriculture demeure le moteur dominant de la production. C'est dans l'articulation de cette agriculture au marché qu'il faut chercher la dynamique du système économique des campagnes maskoutaines au début des années 1830 . 
Le village est le lieu où se concentrent les pouvoirs et les échanges entre la société rurale et le monde extérieur. Durant le premier tiers du XIXe siècle, la croissance démographique et l'intensification de la production agricole ont favorisé l'essor de l'artisanat tandis que l'émergence d'un prolétariat rural témoigne d'une division sociale de plus en plus prononcée de la société paysanne.

La terre maskoutaine est d'abord la propriété des paysans et l'emprise directe des notables sur celle-ci demeure relativement faible au début des années 1830. Dans son ensemble, la propriété paysanne a fort bien résisté au morcellement dans les terroirs plus anciens de la seigneurie et on assiste même à une consolidation des patrimoines fonciers durant le premier tiers du XIXe siècle. Par contre, les nouveaux terroirs subissent directement les contrecoups de la pression démographique. La multiplication des petites propriétés foncières est l'indice d'un accès de plus en plus restreint aux terres neuves et, surtout, d'une vive concurrence entre les nouveaux colons.

Globalement, la hiérarchie des fortunes mobilières respecte l'ordre social traditionnel. Les notables, en particulier le seigneur, le curé et les marchands ruraux, occupent les échelons supérieurs, mais, aux échelons intermédiaires, les clivages sont moins nets. D'après leurs actifs mobiliers, les artisans et les cultivateurs constituent en quelque sorte la couche moyenne de cette société rurale. Cependant, à l'intérieur de ces deux groupes, la diversité des conditions économiques est particulièrement prononcée. Ainsi, les actifs mobiliers des cultivateurs plus aisés révèlent des niveaux de vie comparables à ceux d'une partie de la notabilité rurale, tandis que les actifs mobiliers des moins nantis renvoient à la pauvreté, voire à l'indigence de la masse des journaliers.

La croissance des actifs mobiliers de la paysannerie maskoutaine durant le premier tiers du siècle infirme, à l'échelle régionale, l'une des conséquences présumées de la crise rurale: l'appauvrissement global de la paysannerie. En effet, nous avons observé une aisance accrue chez les paysans des terroirs plus anciens, mais les inégalités économiques sont partout présentes et reflètent la division sociale immanente de la paysannerie. L'émergence d'un prolétariat rural constitue l'une des facettes de cette réalité, tandis que la pauvreté relative des terroirs de peuplement récent représente l'autre facette. Globalement, la société rurale maskoutaine est au rendez-vous de la croissance économique durant le premier tiers du XIXe siècle. Mais ce type de développement renforce les inégalités économiques et sociales, d'autant plus que les mécanismes traditionnels de la reproduction sociale de la paysannerie sont désormais perturbés par l'amorce d'un blocage agraire. Enfin, la notabilité rurale est apparemment le premier bénéficiaire des transformations des campagnes. Au cours de cette période, elle accroît sa fortune, son pouvoir et son prestige au sein des communautés rurales. 